\title{
BRUITS ANONYMES ET DE VOISINAGE
}

l est courant d'opposer les "quartiers populaires traditionnels ", où l'interconnaissance serait de règle, aux "quartiers résidentiels" dont les habitants chercheraient plutôt à éviter leurs voisins. Qu'en serait-il alors des nouveaux "quartiers-villages", où les gens «se connaissent et se parlent ${ }^{1}$, et dont, cependant, la composition sociale, en cours de mutation, n'a plus grand chose de populaire? De fait, le voisinage et les relations de proximité dans l'environnement d'habitat peuvent être vécus aussi comme une contrainte, une entrave à la liberté; ce dont certains tentent de se soustraire en s'efforçant de ne pas sortir de l'anonymat. Et cette différence d'attitude ne peut être réduite uniquement à une différence de milieu social.

C'est ce qui nous est apparu à partir d'une recherche dans un domaine qui semblait - au départ - éloigné de ces préoccupations : celui des réactions aux bruits en milieu d'habitat urbain, et notamment aux gênes, dites "subjectives $»^{2}$. Nous avons relevé une grande variabilité - interindividuelle et intergroupes - de perception de ces bruits, cette variabilité se trouvant, le plus souvent, en relation d'étroite interdépendance avec une grande variabilité d'aspirations en matière de sociabilité de voisinage : allant de la recherche de contacts de proximité à l'évitement de ces derniers.

\section{Anonymat urbain et contexte de voisinage}

Selon la définition du Petit Robert, est anonyme : ce qui est «impersonnel, neutre»; «l'auteur (qui) n'a pas laissé son nom ou l'a caché»; ou encore, ce "dont on ignore le nom", notamment la "foule anonyme». Cette dernière situation peut être vécue par le citadin comme une "pellicule protectrice" (Pétonnet, 1987) de l'individu et de sa vie privée et être appréciée comme telle : en effet, selon cet auteur, les lieux d'anonymat urbain semblent préserver des espaces de liberté individuelle accrue, dans la mesure où ils permettent d'échapper au contrôle social des milieux d'interconnaissance. Mais on peut penser aussi qu'il existe d'autres attitudes possibles, de la part des habitants des villes - eux-mêmes très diversifiés, et parfois venus récemment de la campagne. Ainsi, à l'inverse d'être vécu comme une "pellicule protectrice», l'anonymat peut référer aussi à une impression d'isolement et de solitude; la notion d'anonymat évoquant alors ce qu'on pourrait appeler une impression de "vide social». Et ceci renvoie à un autre type de représentation de la modernité urbaine anonyme : celle de l'atomisation et de la rupture du lien social.

Ainsi, on peut considérer les espaces publics urbains comme des lieux qui offrent des conditions favorables au maintien de l'anonymat; ceci en raison du caractère éphémère des situations de co-présence possibles des individus perdus dans la foule ${ }^{3}$. Mais, en est-il de même en milieu de voisinage?

Il en est tout autrement, en effet, lorsqu'on se trouve dans un lieu où son adresse est plus facilement repérable. Et ceci se vérifie particulièrement lorsque l'on se rapproche du domicile : notamment, par rapport aux voisins. Un voisin, en effet, n'est jamais aussi totalement anonyme qu'un inconnu croisé dans la rue, ne serait-ce qu'en raison de la grande proximité de son lieu d'habitat et de sa durée; ce qui limite, de façon drastique, les échappatoires possibles. Réciproquement - et quoi qu'on fasse - on n'est jamais totalement anonyme, non plus, pour ses voisins. Car tout incline à ce que cette situation d'anonymat de voisinage - déjà plus limitée, au départ, que dans l'espace public - ait tendance à se rétrécir de plus en plus avec le temps : au début de leur installation dans un nouveau domicile, il

\footnotetext{
1. C'est en ces termes, par exemple, qu'un magazine comme l'Express présente un reportage sur le quartier actuel de "La Butte aux Cailles » à Paris, XIlle arrondissement (L'Express, n 2594 du 22 au 28 mars 2001)

2. C'est-à-dire le vécu, représenté et relaté, de ces bruits, par les habitants; ceci par opposition aux gênes dites «objectives », c'est-à-dire aux effets physiologiques et/ou comportementaux des bruits, tels qu'ils sont observés et mesurés par des tiers extérieurs (le plus souvent en laboratoire et indépendamment de la façon dont ils sont vécus consciemment par les intéressés).

3. Ainsi, selon Colette Pétonnet, I'anonymat peut être vécu comme une «pellicule protectrice » qui rend possible l'entrée en contact avec des inconnus : parce qu'on est mutuellement anonymes, ce qui rend libre de ne plus jamais se revoir (Pétonnet, 1987)
}

Les Annales de la recherche urbaine $n^{\circ}$ 90, 0180-930-IX-01/90/p. 93-102 (C) METL 
peut arriver que les voisins soient indifférenciés avec l'homme - totalement anonyme - de la rue; mais ils ne tardent pas à être identifiés, ne serait-ce qu'en tant que voisins.

En d'autres termes, les voisins se trouvent, spatialement et temporellement, en situation privilégiée par rapport à la sortie de l'anonymat : en raison de la multiplication, avec le temps, des probabilités de co-présence dans les espaces communs de voisinage; en raison d'échanges d'informations possibles - réelles ou

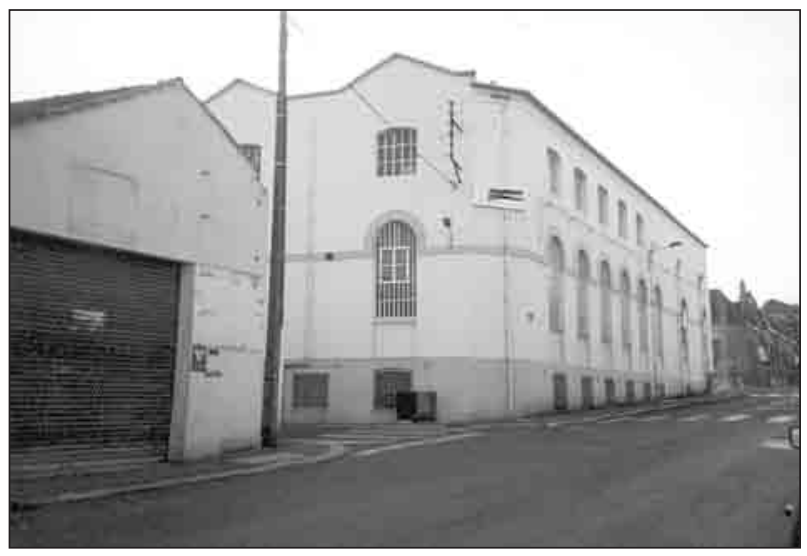

Imprimerie bruyante et maisons attenantes.

imaginaires - entre voisins tiers (les commérages). Mais aussi en raison du poste privilégié de perception et d'observation qu'ils occupent par rapport au domicile : par la vue, éventuellement par l'odorat et surtout par l'ouïe. Car, si les murs du domicile (les rideaux, etc.) peuvent soustraire efficacement à la vue, ils ne protègent pas toujours de l'écoute des sons et des «bruits». Et dans ces cas, il est beaucoup plus difficile - sinon impossible - de trouver une parade. Ce qui revient à dire qu'en allant jusqu'à faire échec, dans certains cas, aux murs-refuges du domicile, les bruits perçus peuvent représenter, en fait, une intrusion supplémentaire. Et cette intrusion peut donner accès à des sphères encore plus intimes de la vie privée et/ou de celle de ses voisins; intimité que les murs du logement ont «normalement" pour fonction de protéger.

On pressent alors, dans ce contexte, la relation privilégiée qui est susceptible de s'établir entre la perception des bruits et les sociabilités de voisinage, dans leur relation à l'anonymat, selon que l'on cherche à préserver son intimité ou, inversement, à pénétrer l'intimité d'autrui.

\section{L'enquête sur les réactions aux bruits}

De fait, c'est l'étude différentielle des réactions "subjectives" à des bruits peu intenses, en milieu d'habitat urbain, qui avait fait initialement l'objet de notre investigation, de tels bruits - moyens et faibles - étant susceptibles d'être plus particulièrement investis en significations symboliques ${ }^{4}$. Aussi, nous avons cherché à identifier ces différentes significations, à partir de l'étude des perceptions. Ceci nous a conduit au choix de deux unités de voisinage exposées à de tels bruits et très diversifiées quant à leurs caractéristiques.

Les gênes conscientes, dites "subjectives", - ou leur absence - étaient appréhendées comme des «faits totaux", à la fois sociaux (Mauss, 1950), et psychologiques (Gurvitch, 1963)5. L’enquête, in situ, avait été réalisée à partir d'une analyse de contexte : par observation directe, mesure de sons, recueil de documents. Elle comprenait une série d'entretiens non directifs, de longue durée, auprès des habitants; ce n'est qu'en fin d'entretien que le discours des enquêtés était recentré sur des thèmes plus précis d'investigation. Les «significations» distinctives, ainsi dégagées, telles qu'elles s'exprimaient à travers les entretiens, ont été identifiées à partir de la méthode des oppositions commutatives de l'anthropologie structurale, ellesmêmes inspirées de la linguistique structurale (LeviStrauss, 1958). Afin de ne pas induire les enquêtés, les entretiens partaient d'une consigne de départ très large : le cadre de vie, en général, des habitants; le thème des "bruits" n'était jamais abordé par l'enquêteur. Cette méthode indirecte (dite «en entonnoir»), a rendu possible la mise en évidence de plusieurs types de problèmes sociaux, d'ordre général et local, dont certains - les problèmes de sociabilité en rapport avec l'anonymat - semblaient se trouver en relation avec le "bruits» et les autres nuisances des sites. De même, l'univers des phénomènes sonores, perçus, représentés et vécus par chaque enquêté, était plus

4. En réalité, la perception des bruits - et les gênes «subjectives » qu'ils sont susceptibles d'entraîner - dépendent beaucoup plus des significations qui leur sont prêtées que de l'intensité sonore ou d'autres caractéristiques acoustiques. Car, il n'existe aucun critère acoustique qui permette de distinguer un bruit d'un son : un bruit n'étant - selon les acousticiens - «qu'un son désagréable » (Kryter, 1985); c'est à dire un son perçu « subjectivement » comme déplaisant. Ceci peut se vérifier même dans le cas de phénomènes acoustiques très intenses, nocifs pour l'oreille. Ainsi, la musique contemporaine et la musique "disco » peuvent être considérées comme des bruits par les uns et comme de la musique agréable par les autres, ceci même lorsque cette dernière est très intense et nocive pour l'oreille. On peut citer aussi, parmi bien d'autres exemples, le cas du motocycliste qui fait pétarader son engin par plaisir, malgré - ou plutôt en raison - de l'intensité du bruit; ou l'exemple du bruit de la goutte d'eau du robinet mal fermé, bruit qui peut gêner et même empêcher de dormir - y compris ce même motocycliste. Mais c'est encore plus vrai dans le cas des «bruits» qui ne sont pas nocifs pour l'individu «objectivement », indépendamment des effets «subjectifs» de gêne qu'ils pourraient avoir et de leurs répercussions indirectement "objectives» (effets de stress etc.). Et c'est, a fortiori, le cas lorsque les «bruits» sont faibles et ne se distinguent en rien, acoustiquement, de la multiplicité des autres phénomènes sonores de l'environnement - sinon par la signification qu'on leur prête. En effet, nous sommes soumis à ces phénomènes, sans y prêter, le plus souvent, attention.

5. Concept élaboré par Georges Gurvitch, en prenant modèle sur le concept de «Fait social total» de Marcel Mauss (La vocation actuelle de la Sociologie, t. 1) 
systématiquement exploré et approfondi en fin d'entretien $^{6}$.

Une telle perspective et une telle démarche se démarquaient totalement des approches habituelles - psychoacoustiques et psycho-sociales - des gênes aux bruits. Car ces approches sont le plus souvent de type expérimental, ou bien elles donnent lieu à des enquêtes par questionnaires - souvent fermés -, qui font totalement abstraction des «sites» et de leurs particularités contextuelles. Ce qui conduit à construire des objets fictifs "gênes" aux bruits -, sans rapport avec les "gênes" réellement vécues. Alors qu'en réalité, les différentes significations dont sont investis les bruits sont inscrites concrètement dans le champ réel de la vie quotidienne des habitants gênés; et notamment, par rapport au contexte spécifique de sociabilité propre à la situation de voisinage, notamment par rapport à l'anonymat.

\section{La dynamique de transformation des sites d'enquête}

Les deux sites d'enquête retenus présentaient des traits contrastés, tant par rapport à leur exposition aux nuisances, que par rapport à leur type d'habitat ainsi que par rapport à leur dynamique sociale d'évolution. Très schématiquement, cette évolution allait plutôt dans le sens d'un délitement des relations de sociabilité, du fait de l'arrivée progressive de nouveaux habitants, ainsi que par l'existence d'un clivage social et relationnel entre les anciennes et les nouvelles populations.

\section{L'habitat et l'exposition aux nuisances}

Le premier site, situé dans une ville moyenne de l'Aisne, comprenait quatre rues bordées, d'un seul côté, de petites maisons de type semi-pavillonnaire, à murs mitoyens, caractéristiques dans la région; des habitations qui, de l'avis de tous, isolaient bien des bruits des voisins. Ces rues entouraient une imprimerie travaillant la nuit. Considérée comme bruyante, celle-ci avait fait l'objet de nombreuses plaintes et pétitions auprès des services des Établissements classés. Les bruits entendus, certains intermittents (en provenance des presses et des rotatives), d'autres continus et continuels (en provenance de ventilateurs), ne dépassaient généralement pas les 55 décibels, lorsqu'ils étaient perçus dans les rues adjacentes. Et leur valeur était bien moindre à l'intérieur des maisons; au point qu'il arrivait que certains des nouveaux habitants ne s'étaient même pas rendu compte de leur existence. À ces bruits de machines, venaient s'ajouter celui des ouvriers que l'on entendait plus particulièrement au moment de la relève des équipes, à cinq heures du matin et à onze heures du soir (bruits de conversations, de radios, claquements de portières de voitures, allées et venues de véhicules - dont des camions de livraison). L'imprimerie, une multinationale non polluante, avait son siège social à l'étranger et apparaissait comme totalement étrangère au quartier. On n'en connaissait - avant les plaintes - ni la direction ni les ouvriers. Elle présentait une apparence soignée qui la distinguait des usines de la région : ceinte de murs blancs bordés de peupliers, dépassant les murs, elle n'avait rien de l'apparence habituelle d'une usine. Elle se distinguait notamment, en tous points, d'une ancienne usine de tissage, dont elle avait pris la place.

Le deuxième site, situé dans une ville moyenne du Forez, s'opposait presque en tous points au premier. La zone d'habitat étudiée se résumait à un grand immeuble rectangulaire, de dix étages et de quatre cages d'escaliers, construit il y a une quinzaine d'années. À l'inverse de ce qui se passait dans le premier site, l'immeuble se trouvait exposé aussi à différentes sources de bruits et de nuisances : perception possible des bruits des voisins, bruits de ventilateurs d'une aciérie, bruits de circulation intenses et élevés, pollutions atmosphériques et poussières sales (en provenance des usines et de la circulation), odeurs d'une rivière-égout à ciel ouvert, mitoyenne de l'immeuble. En revanche, on n'entendait pas de bruits de machines autres que les ventilateurs, ni le bruit des ouvriers. De même, il n'existait aucune rue, ou autre voie de cheminement, permettant d'entendre les bruits de l'aciérie à l'extérieur des appartements?

\section{Arrivée de nouveaux habitants et processus de transformation sociale}

Les populations interviewées comprenaient un éventail assez diversifié de catégories sociales, allant des cadres à des ouvriers et petits employés ${ }^{8}$. Cependant,

6. La recherche, par commutation, des oppositions distinctives existant au sein d'un même entretien, puis entre tous les entretiens d'un même site et, enfin, par rapport à la totalité des deux sites, nous a permis de dégager différentes oppositions d'attitudes, de représentations, de normes, de conceptions et de visions des choses et du monde, ainsi que d'autres dimensions. Ces ensembles, ou conglomérats, s'opposaient à partir de configurations distinctives - à différents types et modalités de réactions à ces «bruits» et à ces nuisances (ou à leur absence).

7. Les fenêtres des appartements donnaient toutes : d'un côté sur l'un des principaux axes de circulation traversant la ville du deuxième site; de l'autre sur la zone industrielle, qui présentait - au loin - un paysage indifférencié d'usines, parfois polluantes. L'une de ces usines, une aciérie plus proche, avait fait l'objet de nombreuses plaintes et pétitions. On se plaignait de ses fumées polluantes et sales (aux couleurs changeantes que I'on pouvait observer), mais surtout de ses bruits de ventilateurs. Pourtant ces bruits étaient très inférieurs aux bruits de circulation, perçus dans les autres parties des appartements. Lorsqu'ils parvenaient au pied de l'immeuble, ils n'étaient pas plus élevés que ceux de l'imprimerie du premier site; et n'étaient pas perçus, non plus, par tous les nouveaux habitants.

8. Les enquêtés du premier site comprenaient un grand nombre de retraités. Parmi ces derniers, on comptait un grand nombre de femmes seules. Notamment en raison de la présence d'un «béguinage» (anciens corons de la région, mis gratuitement à la disposition de vieilles femmes seules de milieu très modeste, par la municipalité de la ville). 
on pouvait les regrouper en deux groupes sociaux distincts par rapport à la dynamique d'évolution des sites : d'une part, une catégorie sociale "moyenne", regroupant des cadres, des techniciens et des ouvriers hautement qualifiés; d'autre part des populations considérées comme modestes par les premières : ouvriers peu qualifiés, petits employés etc. En réalité, on assistait, dans les deux sites, à une dynamique de transformation sociale; mais en direction inverse. Cette dynamique semblait avoir été principalement enclenchée par l'arrivée de nouveaux habitants, souvent plus jeunes et de milieu social différent. Ainsi, dans le premier site on pouvait parler d'une tendance à la revalorisation sociale. On assistait au renchérissement du prix des maisons traditionnelles - de plus en plus convoitées -, et à l'arrivée de nouveaux propriétaires. Ces derniers prenaient progressivement la place des anciens habitants (à la suite de départs en maisons de retraite, de décès sans successeurs, etc.). Dans le deuxième site, le départ - de plus en plus fréquent, aussi - des anciens habitants se traduisait, au contraire, par une dévalorisation sociale et marchande. Les anciens habitants, de milieu plus moyen - et le plus souvent propriétaires -, louaient leurs appartements - trop dévalués pour être vendus - à des personnes de milieux plus modestes. Ces dernières considéraient généralement cette solution comme temporaire. Elles déménageaient fréquemment, dès qu'elles trouvaient à se reloger ailleurs, dans de meilleures conditions.

\section{Le décalage social entre habitants et ses limites}

En réalité, il préexistait encore des infrastructures de sociabilité, plus ou moins tenues, entre les anciens habitants. Ces infrastructures semblaient plus denses chez les anciens habitants du premier site. Ces derniers habitaient parfois celui-ci depuis quarante ans, époque où il existait encore des relations d'interconnaissance étroites, caractéristiques des milieux populaires traditionnels. En revanche, ces infrastructures étaient beaucoup plus lâches chez les anciens habitants - souvent co-propriétaires - du deuxième site : elles se réduisaient souvent à des relations de type utilitaire et fonctionnel. Mais, avec l'arrivée de nouveaux habitants, toujours plus nombreux, on assistait, dans les deux sites, à un phénomène de délitement progressif des relations de voisinage. Un phénomène dont ne pouvait rendre compte le seul fait du décalage social réel ou perçu comme tel - entre ces deux groupes de populations; ni en raison de différences éventuelles relatives aux normes traditionnelles de sociabilité auxquelles ils avaient été soumis auparavant.

De fait, on relevait des manifestations - plus ou moins explicitées - de distance sociale, voire de mépris, entre habitants. Notamment en ce qui concerne les niveaux d'aspiration d'une partie des anciens habitants du deuxième site, interviewés, vis-à-vis des nouveaux, alors que ces derniers aspiraient à s'intégrer aux infrastructures de sociabilité préexistantes, Ceci apparaissait de façon particulièrement explicite dans le deuxième site, en raison de la perception très dévalorisée des nouveaux habitants par les anciens.

Les enquêtés qui explicitaient leur condescendance envers leurs voisins, se référaient, de façon unanime, au manque d'éducation de ceux-ci; des gens considérés comme rustres, "mal embouchés" et qualifiés volontiers d' "asociaux». Il n'en reste pas moins que cette représentation quasi-consensuelle quant au manque d'éducation des voisins tendait à se décliner différemment, selon que les habitants, qui portaient ce jugement, aspiraient ou pas (à de degrés divers) à des relations d'interconnaissance en milieu d'habitat. Ainsi, aux yeux de ceux qui, de façon générale, déclaraient préférer l'évitement des relations de voisinage, le caractère rustre, "asocial» des habitants de milieu plus modeste référait au "sans-gêne" qu'on leur prêtait, à leur absence de précautions, notamment en matière de bruits. Autrement dit, il leur était reproché d'être trop présents. Inversement, pour les enquêtés qui aspiraient à plus de contacts de voisinage, la rustrerie et l'"asocialité" prêtée aux habitants de milieu plus modeste se rapportait principalement, cette fois, à leur caractère peu communicatif, "peu avenant»; ce qui se manifestait notamment, selon eux, par la carence de ces derniers en matière de relations de voisinage. Tandis que les bruits desdites populations "asociales" n'étaient pas considérés comme gênants ou n'étaient même pas perçus, contrairement à ce qui se passait dans les cas précédents.

Nous avons trouvé des différences d'aspiration comparables chez les enquêtés de condition sociale plus modeste, quant à leur désir d'entretenir - ou pas - des contacts sociaux de proximité. Bon nombre d'entre eux, mais quelques-uns, aussi, parmi les enquêtés de milieu plus moyen ${ }^{9}$, avaient gardé en mémoire les relations d'interconnaissance très soutenues qu'ils avaient connues - ou subies - dans leurs lieux d'habitat antérieurs : villages ou petits bourgs, quartiers traditionnels populaires.

Certains en gardaient la nostalgie, et ce n'était pas exclusivement les enquêtés de milieu plus modeste ${ }^{10}$. D'autres n'en retenaient que le côté normatif et contraignant, ce dont ils appréciaient d'être libérés; et ce n'était pas exclusivement les interviewés de milieu plus moyen ${ }^{11}$.

9. C'était le cas, notamment, d'une femme de commerçant ainsi que d'un étudiant en médecine et de sa femme, une maîtresse auxiliaire.

10. Notamment la femme de commerçant ainsi qu'une directrice d'école.

11. On comptait aussi, parmi eux, des ouvriers non qualifiés. 
Ainsi, en dépit des diverses tendances, inflexions et spécificités propres qu'apportaient le milieu social et les expériences de vie qui lui étaient majoritairement associées, ces dimensions ne permettaient pas de rendre compte, à elles seules, des divergences d'aspirations relevées, en matière de relations de voisinage. Sinon en interdépendance avec bien d'autres facteurs comme, par exemple : l'âge ${ }^{12}$; le degré de "mal-être» dans l'isolement social et/ou de demande de sociabilité, ce qui se trouvait, dans bien des cas aussi, en relation avec l'âge et le sexe $\mathrm{e}^{13}$. Mais, comme pour le milieu social et les normes de sociabilité que l'on avait connues, on rencontrait aussi des cas où l'âge et l'isolement social exerçaient un effet inverse ${ }^{14}$. Et ceci nous a conduite à reprendre, en partie, à notre compte les analyses d'auteurs comme B. Matalon (1981), quant aux limites de telles variables "classiques" - dites "objectives" - des sciences sociales, pour rendre compte de l'hétérogénéité des comportements concrets observés ${ }^{15}$; et à nous situer dans le courant "compréhensif» des ces sciences, tel qu'il est défini par ce même auteur, par opposition aux courants explicatifs et causaux ${ }^{16}$. Car, plutôt que de différencier des groupes d'individus à partir de critères sociaux préétablis, nous avons tenté d' «isoler chaque classe de comportement, (et de) l'expliquer par des facteurs qui lui sont propres" (Matalon, 1981, p.161).

\section{Le rejet de l'anonymat et l'acceptation des bruits «humains»}

Certains habitants rejettent la situation d'anonymat excessif, à laquelle ils estiment être confrontés en milieu de voisinage. Cela se traduit par des réactions de gêne et de rejet des bruits "anonymes" qui s'accompagnent, presque dans tous les cas, d'une meilleure tolérance aux bruits susceptibles de participer au l'effacement de l'anonymat (notamment les bruits des voisins). Toutes ces configurations sous-tendent une demande de sociabilité insatisfaite en matière de sociabilité, en général, ainsi qu'en milieu de voisinage, en particulier - aucun clivage n'étant fait entre ces deux types de sociabilité; la sociabilité de proximité tendant à être privilégiée pour des raisons de commodité, étant donné le mode de vie des enquêtés. Mais il convient de distinguer plusieurs sous-groupes, allant des gênes "de conformisme" aux cas d'intolérance extrême aux bruits anonymes, de demande poussée de sociabilité et de rejet angoissé de la modernité.

\section{Les comportements de résistance à l'anonymat}

Il existe, de la part de certains habitants, des tentatives de résistance à la situation ressentie d'anonymisation; ceci, à partir de prises de contacts avec le voisinage et/ou de tentatives de consolidation de relations préexistantes : les bruits, et les gênes qu'ils occasionnent, deviennent alors les prétextes qui facilitent ces contacts. Mais ces contacts n'étaient possibles que lorsqu'il existait déjà des infrastructures de sociabilité. Certains des anciens habitants cherchaient ainsi à consoli-

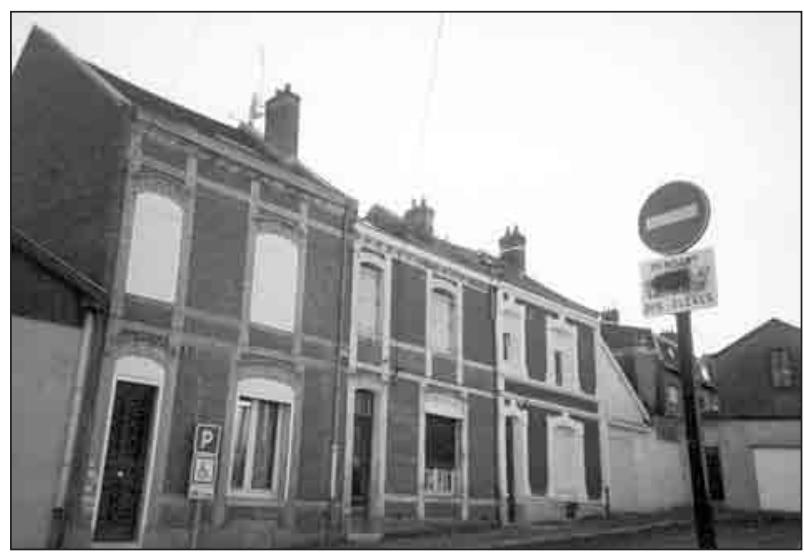

Quelques maisons face à l'imprimerie.

der et à réactiver les liens préexistants, à nouer de nouveaux contacts, ainsi qu'à renforcer leur insertion locale; ceci au moyen d'échanges relatifs aux problèmes du site, en l'occurrence les bruits et les nuisances. C'était plus particulièrement le cas dans le premier site, notamment pour certaines personnes âgées et seules, de sexe féminin et de milieu très modeste. On retrouvait bien que moins souvent - des comportements comparables dans le deuxième site, où les relations entre les anciens habitants étaient, de toutes façons, moins tenues à l'origine que dans le premier. (À titre illustratif, on peut citer, par exemple, le cas de la concierge qui cherchait ainsi à accroître son insertion parmi les copropriétaires).

Dans toutes ces situations, les gênes aux bruits semblaient faibles et peut-être inexistantes. Il s'agissait plu-

12. Indépendamment de leur milieu social, les personnes qui préféraient éviter leurs voisins, étaient plus souvent jeunes; mais pas exclusivement, loin de là.

13. C'était plus souvent le cas, notamment, de femmes d'âge mûr, souvent célibataires, et souffrant de leur isolement.

14. Certains jeunes affichaient des attitudes d'ouverture au contact humain quel qu'il soit; certaines femmes mûres, souffrant de carence en matière de sociabilité, réagissaient, au contraire, par le repli sur soi et l'évitement des voisins.

15. Ainsi, selon B. Matalon, ces variables «ne permettent pas plus que d'autres de définir des groupes vraiment homogènes du point de vue des comportements, même si les distributions de ceux-ci sont susceptibles de varier de façon significative d'un groupe à l'autre» (p. 148).

16. B. Matalon regroupe dans le courant «compréhensif», «aussi bien la sociologie phénoménologique ou l'ethnométhodologie que les tenants de l'individualisme méthodologique » (p. 159); ceci par opposition aux courants « déterministes», qui cherchent à mettre en évidence les relations entre, d'une part, les variables "classiques», "objectives», les "caractéristiques sociales des individus»; d'autre part, leurs « comportements, opinions et autres traits» (p. 158) 
tôt de "gênes" que nous avons appelées de "conformisme». "Gênes» dont le bénéfice secondaire était la convivialité, mais aussi l'élargissement du champ relationnel des personnes concernées à des voisins de milieu social plus élevé, ou/et le renforcement de leur insertion parmi ces derniers. De tels comportements étaient souvent le fait d'habitants de milieux plus modestes. Tandis que les milieux plus aisés assumaient plus souvent le rôle de leaders de plaintes et de pétitions.

\section{Les attitudes «voyeuristes» envers les voisins}

Certaines personnes manifestent ce que nous avons appelé des attitudes de "voyeurisme». Elles sont à l'affût de la présence des voisins, dont elles vont même jusqu'à rechercher les bruits. Inversement, les mêmes manifestent des réactions de gêne particulièrement extrêmes aux bruits de circulation et, parfois plus encore, aux bruits des usines. Elles se disent particulièrement gênées par les bruits des machines; mais aussi, parfois - bien que dans une moindre mesure -, par le bruit d'un personnel «anonyme» qui les ignore. Les bruits d'usines sont, alors, d'autant plus exécrés que ces dernières prennent spatialement la place de voisins, que - selon leurs propres déclarations - ces enquêtées auraient souhaité avoir.

Ainsi, l'anonymat peut être vécu comme un véritable traumatisme par des personnes frustrées dans leur demande de relations de sociabilité sur le lieu d'habitat. Cela peut se traduire alors par de la souffrance intense et l'accablement devant des bruits "anonymes", qui évoquent la situation de trop grand vide social à laquelle elles se sentent confrontées. Cet état de choses, devant lequel on se trouve totalement désemparé et impuissant, est attribué à une situation d'anonymat excessif de voisinage. Ceci, d'autant plus qu'il s'agit, parfois, d'une situation vécue tout à fait nouvelle. C'est le cas, notamment, de certaines personnes transplantées de la campagne ou de petits immeubles populaires traditionnels, dans le deuxième site. En butte à l'ostracisme des anciens habitants, elles se trouvent dans une situation d'anonymat total. Or, ces enquêtés avaient toujours vécu dans des milieux de voisinage d'interconnaissance, milieux dans lesquels elles avaient été parfaitement insérées auparavant. À leurs yeux, les relations de convivialité, de "solidarité» et "d'entraide», qu'elles avaient connues, l'emportent largement sur les inconvénients du contrôle social, propre à ces milieux.

Ce sous-groupe tendait à indifférencier, en fait, les bruits de circulation et les bruits des usines dans une même dimension de rejet; ce qui renvoie à une catégorisation de même type dans la classe des «bruits anonymes». D'où, dans le deuxième site, la propension à être beaucoup plus gêné par les bruits de circulation que par les bruits des usines - beaucoup plus faibles. Or, c'est tout à fait l'inverse pour le sous-groupe suivant.

\section{Le rejet angoissé de la modernité et de l'artifice}

Pour certaines personnes, les notions d'anonymat de voisinage et de vide social tendaient à être intégrées dans une vision plus globale et structurée d'un monde moderne menaçant et aliénant. Au sein d'un tel monde, l'anonymat ne représentait plus, alors, que l'une des dimensions significatives - parmi d'autres d'une situation plus globale de déshumanisation. Aux yeux de ces personnes, la situation présente s'opposait en tous points, à la vision d'un monde passé, idéalisé, qu'ils avaient connu dans leur enfance, au sein d'un environnement "écologique», convivial et harmonieusement familial.

Les individus concernés faisaient des fixations préférentielles extrêmes sur les bruits des usines. Au point, que dans le premier site, une directrice d'école avait avancé l'âge de sa retraite pour quitter son logement de fonction, exposé au bruit de l'imprimerie. De même, deux personnes du deuxième site avaient déménagé pour cette seule raison (l'une d'entre elles, une ouvrière qualifiée, entendait encore le bruit des ventilateurs de l'aciérie, auquel elle n'était pourtant plus exposée).

De façon générale, l'ensemble de ces trois catégories de personnes gênées par les bruits "anonymes" et recherchant les bruits des voisins - était exclusivement composé de femmes : plutôt d'âge mûr, sans profession, ou/et vivant seules, ou/et s'estimant délaissées par leur conjoint, ou/et par leurs enfants (déjà grands). Elles auraient souhaité combler leur vide relationnel en établissant des liens avec leurs voisins (avec lesquels elles ne se sentaient nullement en décalage relationnel, quand bien même il existait un décalage social). Beaucoup avaient vécu auparavant dans des lieux où l'interconnaissance entre voisins faisait figure de norme; une norme à laquelle ces enquêtées adhéraient parfois de façon explicite.

\section{Le rejet des relations de sociabilité entre voisins}

On peut faire état d'une tendance à l'opposé des attitudes précédentes. Dans ce cas, les habitants cherchent à préserver - autant que faire se peut - une intimité jugée menacée par leurs voisins et expriment une meilleure tolérance aux bruits "anonymes» qui, généralement, ne gênent pas. Ils vont parfois même jusqu’à apprécier de tels bruits, parce qu'ils prennent la place de voisins potentiels. Mais cette tendance s'accompagne de gênes et de rejet des bruits "non anonymes", et plus particulièrement lorsqu'il s'agit des 
bruits des voisins. Car, en dévoilant davantage l'intimité d'autrui, ces derniers bruits contribuent à faire échec aux relations de distanciation du voisinage et d'anonymat que l'on cherche à préserver. Ceci d'autant plus que de tels bruits contribuent, en fait, à faire prendre conscience du caractère auditivement perceptible de ses propres bruits et donc de sa propre intimité, par le même voisin que l'on entend. Le bruit de ce dernier vient, ainsi, rappeler constamment que l'on ne peut échapper soi-même à celui-ci. Et, dans les cas extrêmes, ceci paraît d'autant plus évident - et générateur de gênes - que les bruits émis sont faibles, tout en étant quand même perceptibles.

Les cas extrêmes peuvent être caractérisés par ce que nous avons appelé des attitudes "d'espionnite». Ces personnes, qui évitent les relations de voisinage, vont parfois jusqu’à apprécier les bruits de machine des usines. Ceci dans la mesure où le voisinage de ces dernières et de leur personnel - aux bruits humains «anonymes" - occupe la place de voisins potentiels que l'on redoute et dont on exècre les manifestations sonores, même lorsqu'elles sont très faibles.

Autrement dit, l'anonymat est recherché dans la mesure où il joue le rôle de "pellicule protectrice» de la vie privée et de la liberté d'action individuelle. Les personnes concernées se caractérisent, essentiellement, par leur relatif individualisme; par leur souci de préserver par l'anonymat - une liberté individuelle, qu'ils jugent menacée.

Mais, sous-jacent à ces attitudes, on trouve parfois le sentiment d'être différent de ses voisins que l'on dévalorise. Les enquêtés de ce type se trouvent donc en décalage par rapport à leur voisinage. Ce décalage peut être d'ordre socio-culturel : par rapport à des voisins de milieu plus défavorisé. Mais le décalage socio-culturel ne suffit pas, à lui seul, pour rendre compte de l'appartenance au type, souvent extrême, décrit ici ${ }^{17}$. Les décalages observés dans ce groupe peuvent être de tous ordres : différences de générations, d'idéologies, de modes et de styles de vie; y compris des représentations fantasmatiques dévalorisantes qui renvoient, parfois, à ce que l'on pourrait appeler du «bovarisme» (on peut citer le cas d'un instituteur célibataire qui se juge d'avant-garde et méprise son voisinage : des proches du même milieu que lui - dont des membres de sa famille, et sa propre sœur). De fait, ces enquêtés rejettent d'autant plus leurs voisins, que ces derniers sont ressentis comme présentant une image d'eux-mêmes à laquelle ils refusent de s'identifier. Ainsi, dans les cas de gêne extrême le voisin représente une menace, non seulement pour sa liberté, mais aussi pour sa propre identité.

On rencontre plus souvent ces attitudes chez les enquêtés de milieu socio-culturel plus élevé, et/ou d'origine urbaine, et/ou plus jeunes. Mais c'est plus particulièrement le cas, aussi, des personnes qui ont

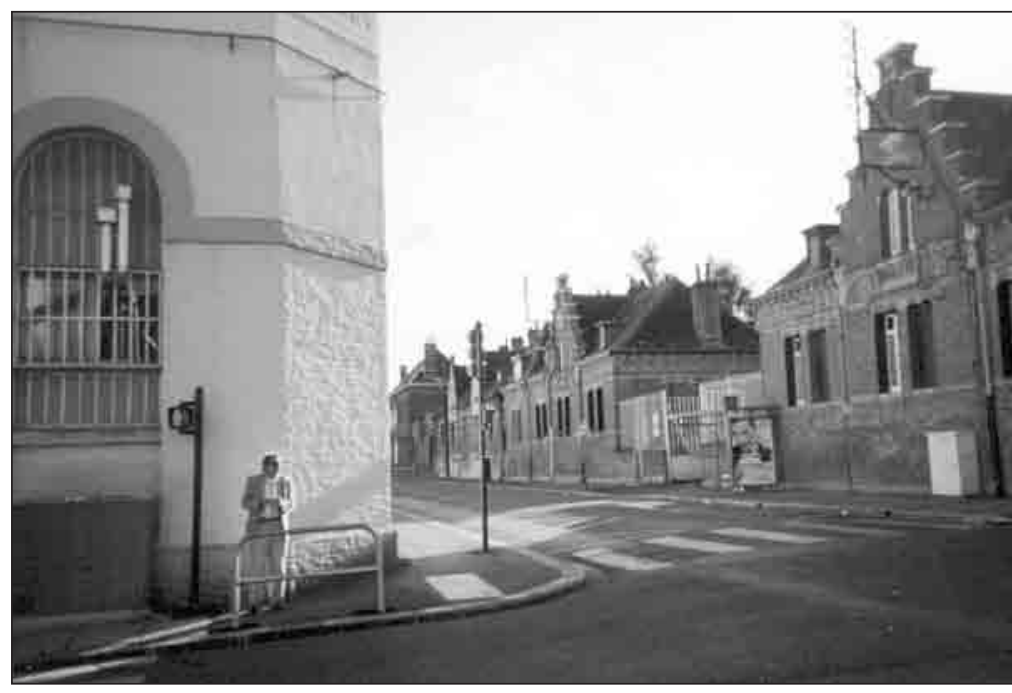

Maison et «béguinage » faisant face à l'usine bruyante.

souffert antérieurement, ailleurs, de situations de voisinage plus contraignantes : notamment certains interviewés - plus jeunes - en provenance de milieux populaires traditionnels d'interconnaissance. Autrement dit, les normes d'interconnaissance entre voisins, qu'ont connues certains, jouent, cette fois, un rôle inverse de repoussoir. En matière de normes de sociabilités, ce dernier cas de figure semble exprimer une tendance à la résorption des anciennes spécificités du milieu de voisinage, au profit du modèle du milieu indifférencié des espaces publics urbains.

\section{Le rejet indifférencié des bruits et de l'environnement}

L'indifférenciation des bruits peut signifier aussi que l'on redoute ces derniers, en tant que menaces de conflits de voisinage. Tous les bruits perçus dans l'environnement - bruits de voisins ou d'usines -, sont alors considérés de la même façon : comme des supports éventuels de conflits. On prête donc de façon égale ce pouvoir à tous les bruits.

Les enquêtés concernés tentent alors d'échapper à ces menaces de conflits, par la distanciation et l'anonymisation. Ou bien ils y succombent; parfois, en raison de la révolte que peut provoquer, chez certains, le sentiment de l'anonymisation de soi par l'autre. De telles réactions se rapportent, tout aussi bien, aux bruits des voisins qu'à d'autres fauteurs de troubles du voisinage : notamment les bruits des usines voisines. Dans ce cas, les bruits de circulation comme les bruits des machines irritent : surtout dans la mesure où ils réferent, en fait -

17. Ainsi, les anciens habitants du deuxième site, qui manifestent de l'ostracisme à l'égard des nouveaux, ne relèvent pas tous de cette catégorie (certains sont plutôt de type "conflictuel»; d'autres cas relèvent, en fait, des gênes de « conformisme») 
de la part des enquêtés redoutant par dessus tout «l'espionnage» et le bruit de ces derniers, mais tolérant ou même appréciant les bruits "anonymes"; des stratégies symétriques et inverses de dévoilement de l'anonymat des voisins, de la part de personnes qui allaient jusqu'à rechercher leurs bruits et qui redoutaient, par dessus tout, les bruits "anonymes" qui évoquaient, pour elles, le vide social. Le rejet de l'anonymat par certains, pouvait s'accompagner aussi de tentatives de résistance à celui-ci, notamment de la part de ceux qui se saisissaient des bruits comme prétexte d'entrée en contact : afin d'en nouer de nouveaux et/ou de consolider les anciens; et/ou afin d'établir ou de conforter une position de leadership, à travers la prise en charge d'actions collectives de plaintes et de pétitions. De même, il existait une gamme d'autres réactions : allant de la révolte à l'accablement : face, notamment, aux bruits "anonymes" des usines; ces dernières étant jugées elles-mêmes "anonymes". Ces réactions s'accompagnaient, alors, de réactions de même type, par rapport à ce qu'on vivait comme une situation insupportable - et parfois entièrement nouvelle - d'atomisation sociale et d'anonymisation.

En d'autres termes, il existait différents types d'ordonnancement des bruits et des sons et de leurs sources - humaines ou "anonymes» -, selon qu'ils étaient perçus comme plus ou moins "anonymes». Et ces types se trouvaient en relation de correspondance symbolique, avec des modes de relations distincts à l'anonymat et aux sociabilités de voisinage. Ces liens de correspondance ont pu être établis en raison de l'existence d'oppositions distinctives, relatives aux réactions aux "bruits» et aux phénomènes sonores. Ces oppositions se trouvant en relation d'isomorphisme (Levi-Strauss,
1958) avec ce qui peut être interprété comme des oppositions distinctives de réactions aux processus "d'anonymisation" des sites. Mais, plus généralement, on peut parler d'oppositions de réactions qui se rapportent aux contraintes spécifiques des situations de voisinage, en matière de sociabilités. Car, on peut parler du caractère privilégié de ces situations par rapport au dévoilement de la vie privée et de l'anonymat. On peut parler du caractère privilégié des bruits et des sons, entendus au domicile, en tant qu'instruments et expression de ce dévoilement (les bruits des voisins) - ou, au contraire, de son non-dévoilement (les bruits "anonymes»). En d'autres termes, à travers un problème vécu en milieu d'habitat urbain, celui des gênes "subjectives" aux «bruits» - objet premier de notre investigation -, nous avons été renvoyée à un autre "palier en profondeur" (Gurvitch, 1963, t. I) de la réalité sociale, les sociabilités dans leur relation à l'anonymat.

Cette approche, où l'anonymat urbain s'est trouvé jouer un rôle d'analyseur privilégié, a laissé entrevoir aussi quelques cas-types qui sont peut-être caractéristiques du milieu urbain contemporain : les espaces publics investis de normes latentes d'anonymat et d'absence de contact; les espaces de voisinage en milieu populaire traditionnel, investis de normes d'interconnaissance contraires à l'anonymat; les espaces de voisinage en situation intermédiaire entre ces deux cas de figures. Ces espaces intermédiaires, aux normes de sociabilité encore indéterminées de façon consensuelle, sont le théâtre et l'enjeu d'affrontements entre deux systèmes normatifs dont la dynamique d'évolution semble indiquer une tendance à une anonymisation croissante.

Tatiana Guélin

\section{RÉFÉRENCES BIBLIOGRAPHIQUES}

Boas F., (1916), "Tsimshian mythology», 31 th. Arbae, Washington, D.C.

Boudon R. (1997), Cognition sociale et sciences sociales : la dimension cognitive dans l'analyse sociale, Paris, PUF.

Brown P., Mauffette M., (1999), "Anthropologie cognitive» Anthropologie et sociétés, vol. 23, n³, p.91-119.

Chiva I., (1962), «Étude effectuée dans la région de Bagnolssur-Cèze, à la suite de l'implantation du centre de Marcoule", in : Utilisations de l'énergie nucléaire (aspects scientifiques, techniques et humains), Paris, Masson, p. 415-424.

Chiva I., (1970), "Imagination collective et inconnu», in : Pouillon Jean, Maranda Pierre (éd.), Échanges et communications : mélanges offerts à Claude Levi-Strauss à l'occasion de son 60e anniversaire, La Hague, Paris, Mouton, p. 162-168.

Clément F., (1999), «La sociologie cognitive : une bien étrange croyance?", Les Cahiers internationaux de sociologie, vol. 107, p. 389-404.

Guelin T., (1992), Bruits de l'environnement et vie quotidienne: un champ et une méthodologie nouvelles de recherche? Pour une approche holistique et transdisciplinaire des réactions d'intolérance vécues aux bruits, Rapport multigr.

Guelin T., (1993), Enquête sur les gênes aux bruits industriels urbains : positions théoriques et méthodologie, Rapport multigr.

Guelin T., (1996), "Pour une approche anthropologique des gênes «subjectives» aux bruits urbains", Méridiès, n 19.20/94, p. 51-85.

Gurvitch. G., (1957), La vocation actuelle de la sociologie, Paris, PUF, (3e éd., 1963), t.1.

Haudricourt A.-G., (1964), «Nature et culture dans la civilisation de l'igname : l'origine des clônes et des clans", L'Homme, n'IV (1), p. 93-104.

Jodelet D., (éd.), (1989), Les représentations sociales, Paris, PUF, (2e éd., 1991). 
Kryter K.-D., (1985), The effects of noise on man, London, Academic Press inc.

Levi-Strauss C., (1950), "Introduction à l'œuvre de Marcel Mauss ", in : Mauss M., Sociologie et anthropologie, Paris, PUF, (1973) (5e édition).

Levi-Strauss C., (1958), "La geste d'Asdiwal", Annuaire de l'École pratique des hautes études, Paris, section des sciences religieuses.

Matalon B., (1981), «La psychologie et l'explication des faits sociaux", L'année sociologique, ${ }^{\circ}$ 31, p. 125-185.
Moch A., Moser G., (1997), "Psychologie environnementale : perspectives actuelles ", Psychologie française, vol. 42, $\mathrm{n}^{\circ} 2$, p. 169-174.

Moscovici S., (1988), "Notes towards a description of social representations", European Journal of social psychology, $\mathrm{n}^{\circ} 18$, p. 211-250.

Petonnet C., (1987), «L'anonymat ou la pellicule protectrice», in : Le temps de la réflexion, $\mathrm{t}$. VIII : La ville inquiète, Paris, Gallimard, p. 247-261.

Taylor D.-M., Brown R.-J., (1979), "Towards a more social social psychology?", British Journal of social clinical psychology, $\mathrm{n}^{\circ} 18$, p. 173-180.

Tatiana Guélin est membre du Laboratoire d'anthropologie urbaine du CNRS. Elle se consacre actuellement à l'étude des relations de socialbilité ainsi qu'à la perception sociale des bruits et des nuisances en milieu urbain. Ce dernier domaine est abordé dans une perspective interdisciplinaire et intégratrice du sujet et de son ancrage social. Après plusieurs publications dans ce domaine, elle achève actuellement un ouvrage ainsi qu'une thèse sur ces thèmes. $<$ tguelin@hotmail.com> 